Graf Sponek, Uber den Anbau.... des Ahorns, mit Riicksicht zur Zuckermewinnung. Heidelberg 1811.

v. Werneck, Anleitung zur Ahornzucht mit Rucksicht auf die Gewinnung von Zucker. Marburg 1815.

F. Kait, Gewinnung des Ahornzuckers. Prag 1837.

Ferner Natizen in folgenden Zeitschriften:

Stahl, Forstmagazin, $\bar{x}$, S. 273.

$v$. Mosers Archiv, $X X, 1797$, und $X X 1,1798$

Leonhard, Magazin, $I, 2$, S. 34.

Forst- und Jagdzeitung, 1829, u. a.

\section{Die Jahrhundertfeier \\ des Bestehens der Coast and Geodetic Survey der Vereinigten Staaten von Nordamerika 1916.}

Von Prof. Dr. A. Galle, Berlin-Potsdam, Abteilungsvorsteher im Kgl. Geoditischen Institut.

Fast unbeachtet infolge des Weltkrieges ist die Jahrhundertfeier einer der ältesten Institutionen der Vereinigten Staaten Nordamerikas bei uns vorübergegangen. Wenn wir jetzt auf diese am 5 . und 6. April 1916 in Washington von der Coast and Greodetuic Survey begangene Feier zurückkommen, so liegt der Anlaß dazu in der Veroffentlichung darüber ${ }^{1}$, die der zeitige Superintendent $E$. Lester Jones herausgegeben hat und die ihren Weg über den Ozean hierher vor kurzem gefunden hat. Wir haben Grund genug, uns nachträglich den Glückwünschen anzuschließen, die das für die ganze Welt bedeutsame Unternehmen bei dieser Gelegenheit begrüßt haben und uns über seine Enitwicklung und seine Ziele an der Fiand dieser Gelegenheitsschrift AufschluB zu verschaffen und Rechenschaft zu geben. Denn so verschiedenartig vielfach die Einrichtungen und die Methoden amerikanischer Forschung von den unsrigen sind, so wichtig ist es, sie zu kenmen und sie, wenn nicht in allen Punkten nachzuahmen, loch zu beachten und in manchen Beziehungen zum Vorbild zu nehmen.

Dieser abweichende Charakter tritt sogleich in der Tatsache hervor, daß die genannte Survey dem Handelsdepartement unterstellt ist. Allerlings war sie dies nicht immer. Bis 1834 war sie ron dem Schatzamt (Treasury) abhängig, dann 2. Jahre von dem Marineamt (Navy), dann wieder lange Zeit vom Schatzamt bis 1903; von da ab wurde sie anfangs dem Handels- und Arbeitsministerium (Commerce and Labour), dann 1913 dem Handelsamt (Commerce) allein untergeordnet. Wir sind geneigt, angesichts der großen wissenschaftlichen Erfolge die Coast and Geodetic Survey nach ihrem zweiten Titel als eine Landesvermessung uns vorzustellen, die in den europäischen Staaten in den Händien der Generalstäbe liegt und bei uns noch unter Friedrich dem Großen nur militärischen Zwecken diente. Die Survey aber verdankt ihre Entstehung nicht so sehr oder nicht allein den Rücksichten auf dive Landesverteidigung, sondern noch weit mehr den Inter-

1) Centennial Celebration of the U, S. Coast and Geodetic Survey. Washington 1916. essen des Wrelthandels und des Handels an den Küsten der Vereinigten Staaten. Um die Schifffahrt vor den Unfällen an den besonders im Osten klippenreichen Gestaden zu sichern, die Anlage guter Häfen zu ermöglichen, den Golfstrom zu ihrer Fördlerung zu benutzen, der Fischerei, nicht zum wenigsten dem Austernfang hydrographische Unterlagien zu liefern, entschloß sich der Kongreß auf Grund einer weitsichtigen Entachliebung des damaligen Präsidenten der Republik Jefferson im Jahr 1807, eine Behörde zu schaffen, welche die Erforschung der Küstengewässer zu ihrer Aufgabè haben sollte. Verschiedene Umstände, sodann der Krieg mit England, der von 1812-1814 dauerte, verzögerten die Ausführung des Beschlusses. Aber die einleitendien Schritte waren geschehen, und man hatte den Mann gefunden, der mit hervorragender Begabung und Willenskraft ausgestattet, die Sache von der rechten Seite anzufassen wuBte. Es war ein Schweizer, Ferdinand Rudolf Hapler, der 1770 in Aarau als Sohn eines wohlhabenden Uhrmachers geboren, in Bern sich unter Tralles ${ }^{1}$ ) (geb. 1763 in Hamburg, gest. 1822 in London) in mathematischen Studien ausbildete.

Tralles war der erste, der eine Vermessung der Schweiz vornahm, bei der Haßler ihm beistand, ja sogar eine Grundlinienmessung auf eigene Kosten ausführte. Hierbei sammelte Haßler geodätische Kenntnisse und Erfahrungen. Es mutet wie ein Treppenwitz der Geschichte an, dab ein Ingenieur aus einem Land, das keine Küston besitzt, dazu ausersehen war, die Coast Survey in den Vereinigten Staaten zu begründen, deren kontinentale Küstenlänge, einschlieBlich Alaska, mit allen Einbuchtungen, Inseln und Vorsprüngen auf $150000 \mathrm{~km}$ geschätzt wird. Mit welchem Uberblick er an dic neue Aufgabe herantrat, bezeugt sein Entwurf über die besten Methoden, die dabei angewendet werdien können, der (S. 180-184) aus dem Französischen übersetzt vorliegt. Im Jahre 1811 wurde Hafler nach England gesandt, um die notwendigen Instrumente zu besorgen. Es ist erwähnenswert, daß er eingehende Untersuchungen und Vergleichungen der mitgenommenen Maßstäbe varnahm, wie er wohl z. T. durch Trialles, der an den Beratungen für die Einführung des Mretermakes in Paris teilgenommen hat, in die Methoden der Maßvergleichung eingeführt und mit den beteiligten Pensönlichkeiten bekannt geworden ist.

Ja, unter seiner und seiner Nachfolger Auf sicht war das gesamte Maß- und Gewichtswesen der Voreinigten Staaten der Coast Survey unterstellt, bis dann 1901 ein eigenes Bureau of Standards ins Leben gerufen wurde. Ein Bericht von 1832 stellte die Grundsätze auf, die Haßler zur Reformierung der Maßangelegenheiten für die Zollämter uøw. gab.

Als er 1815 in Washington ankam, brachte er \footnotetext{
Berlin.
} 
die reichste Sammlung nicht nur von Instrumenten, sondern auch von wissenschaftlichen Büchern mit, die jemals über den Ozean gekommen war. Im Jahre 1816 trat die Survey zuerst in Tätigkeit, tatsächlich aber fand im folgenden Jahre eine Unterbrechung bis zum Jahre 1832 statt, während deren Grenzvermessungen durch Ingenieuroffiziere, auch unter Haßlers Mitwirkung, ausgeführt wurden.

Nach seinem Tode sind auf Haßler 10 Männer als Superintendenten gefolgt, deren Namen zum großen Teil in der Geschichte der Wissenschaft fortleben werden: 1843-1867 Bache, 1867-1874 Benjamin Peirce, 1874-1881 Patterson, 1881 bis 1885 Hilgard, 1885-1889 Thorn, 1889-1894 Mendenhall, 1894-1897 Duffield, 1897-1900 Pritchett, 1900-1915 Tittmann, 1915 Jones. Aber neben ihnen wirkten noch andere Männer, deren Namen in der Welt nicht minder bekannt geworden sind, wir erinnern nur an Schott, Hayford und Bowie und an Kapitän Talcott, der 1834-1835 als Mitglied des Ingenieurkorps an der Grenzvermessung zwischen Ohio und Michigan teilnahm; ferner war Peary der Survey zum $\mathrm{Z}$ wecke der Beobachtung der arktischen Gezeiten zugeteilt.

Um nun die Tätigkeit der Coast Survey zu verstehen, müssen wir zunächst ihre ursprüngliche Aufgabe ins Auge fassen.

Die Erforschung der Küsten und Küstengewässer und die Beschaffung der für die Schiffahrt nötigen Hilfsmittel ist allmählich mit der Größe der Schiffe gewachsen. Auf besonderen Schiffsexpeditionen - erst auf Segelschiffen, dann auf Dampfschiffen - wurden anfangs nur durch Lotungen die Verhältnisse des Meeresbodens festgelegt, später wurden durch Schleppen von Drähten, deren Länge bis $6 \mathrm{~km}$ betrug und die durch Schwimmer in gewünschter Tiefe gehalten wurden, Klippen und andere Unebenheiten des Meeresgrundes aufgefunden. Die Lage der durch folgende Lotungen ermittelten Untiefen, Austernbänke und dergl. wurden dann durch Vermessungen in ihrer örtlichen Lage zur Küste bestimmt. Solche Beobachtungen und die Küstenvermessungen selbst wurden in gewissen Zeiträumen wiederholt, um die Karten für die Seefahrer auf dem Laufenden $z u$ erhalten. Es wurden ferner die Orte.für Leuchtfeuer, Baken und Tonnen ausgesucht und festgelegt. Die Untersuchungen der Meeresströmungen und die an denselben Punkten, deren Zahl sich auf 5000 beläuft, öfters wiederholten magnetischen Beobachtungen dienten den $\mathrm{Z}$ wecken der Schiffahrt auf hoher See. Diesen Unternehmungen steht die Erforschung der Meeresgezeiten nahe. Den Untersuchungen von Ferrel 1874 folgten die Arbeiten von Harris, wobei das Gezeitenphänomen, das man anfangs allzu theoretisch als eine die ganze Erde umlaufende Gezeitenwelle aufgefaBt hatte, mehr lokalisiert wurde. Indem man die Wellenbewegungen in mehr oder weniger scharf abgegrenzten Gebieten betrachtete, gelangte man zu Ergebnissen, die mit den tatsächlichen Verhältnissen gut harmonieren.

Bald nach dem Bürgerkrieg (1861-1864) genehmigte der Kongre $B$ eine geodätische Verbindung zwischen den Küsten des Atlantischen und Stillen Ozeans. Es kam hierdurch die gröBte Längengradmessung zustande, die von einem einzigen Staate in einheitlicher Weise unternommen worden ist. Sie hat der Survey selbst für die Methoden der Rekognoszierung, der Triangulation und die Rechenmethoden neue Anschauungen gebracht und TVege gewiesen. Vor allen Dingen hat sie verschiedene gesondert gemessene Gebiete vereinigt. Dieser Bogen in 39 Grad Breite (also gerade in dem Parallel, in dem später die Breitenstationen zur Bestimmung der Schwankungen der Erdachse angelegt worden sind), der mehr als 48 Längengrade umfaßt, hat - und hierin liegt seine universelle Bedeutung - für unsere Kenntnis der Erdgestalt einen bedeutsamen Beitrag ge liefert. Es war eine Folge dieses Unternehmens, daß die geodätische Arbeit der Survey mehr in den Vordergrund trat und äuBerlich zeigte sich dies in ihrer Benennung, indem aus der Coast Survey die Coast and Geodetic Survey hervorging. Gerade die Bedeutung des Ergebnisses für die Erdmessung hatte seine notwendige Ergänzung durch Breitengradmessungen zur Folge. Zunächst wurde jener östliche Bogen gemessen, welcher den Golf von Mexiko mit der Fundy-Bay verband, der die erste größere schräg zum Meridian gelegte Gradmessung ist und der zugleich die getrennten Vermessungsgebiete an der Küste des Atlantischen Ozeans zu verbinden bestimmt war. Mit dieser großen Vermessung ist dauernd der Name von Charles Schott verbunden, der 50 Jahre hindurch bei der Survey tätig, diese Arbeiten und Rechnungen in erster Linie zur Vollendung brachte und dadurch die Einheitlichkeit des ganzen Unternehmens verbürgte.

Je mehr sich in der Folge die groBen Gradmessungen ausdehnten, um so mehr trat das $\mathrm{Be}$ dürfnis hervor, sie auf einen einheitlichen Ausgangspunkt $z u$ beziehen, der ihre Lage und ihre Orientierung auf der Erdoberfläche bestimmte. Dieser als Standard Datum bezeichnete Punkt wurde dann auch von Canada und Mexiko angenommen, so daß seine Benennung seit 1913 in North America Datum abgeändert wurde.

Die zahlreichen astronomischen Bestimmungen, die der Survey verdankt werden, lieferten in Verbindung mit den geodätischen das Material, um jene Ausgleichung der Lotabweichungen zu berechnen, die zur Feststellung der Isostasie der Erdkruste führte. Schwerkraftsmessungen schlossen sich an, die das mit den Lotabweichungen ermittelte Resultat bestätigten.

Wir haben bereits den Namen Talcott erwähnt, der die von dem Dänen Horrebow zuerst angegebene Methode der astronomischen Breitenbestimmung in Aufnahme brachte; die Beteiligung der Coast and Geodetic Survey an der Beobach- 
rung der Breiteuïnderungen auf dem Parallel von Washington durch die beiden Observatorien in Ukiah (Kalifornien) und Gaithersburg (bei Washington), die 17 Jahre in Tätigkeit wareu, brachte dieser Methode ihre wichtigste Verwendurr.

Sodann dürfen wir nicht vergessen, thas die astronomischen Längenbestimmungen bereits unter Bache sich zuerst des elektrischen Telegraphen bedienteu, und daß auch die Längenbestimmungen mit Benutzung der Kabel amerikanischen $\mathrm{Ur}$ sprungs sind.

Die geodätischen Messungen der Grundlinien durch die Survey sind durch eigenartige Apparate ausgezeichnet, von denen derjenige von Woodward, ther die Meßstangen in Eis lagert, als einer der vollkommenston angesehen wird. $\mathrm{Da}_{\mathrm{a}}$ indessen seine Anwendung schwierig und kostspielig ist, so bedient sich die Survey der Stahlbänder, in Abänderung der von dem Schweden Jäderin erfundenen Drahtmessungen, und ist neuerdings dazu übergegangen, das von 'Temperaturïndierungen fast unbeinflußte Invar an Stelle des Stahls für ihre Bänder zu verwenden. Von großer Ausdehnung ist das Nivellementsnetz der Vereinigten Staten, welches abgesehen von seiner praktischen Bedeutung die Höhenlage der trigonometrischen Punkte, der Grundlinien und der Küsten gegeneinander festgelegt hat.

Wenn wir zum Schluß noch einten Rückblick auf die Tätigkeit der Survey von einem allgemeineron Gesichtspunkte werfen, so tritt bei allen Vnternehmungen ein Grundsatz hervor, der auch con einem der Redner bei der Hundertjahrfeier beonders hervorgehoben wurde: Die besten Ergubnisse bei den garingsten Kosten! Uns erscheint es befremullich. wenn die Geschwindigkeit der Jessungen und die geringen Kosten für den Kilometer immer wieder in den wissonschaftlichen Veröffentlichungen erwähnt werden. Offenbar sind diese Bemerkungen in bezug anf die Bewilligungen cles Kongresses von Wichtigkeit, und in clieser. Kontrolle einzelner wissenschaftlicher Arbeiten durch eine parlamentarische Körperschaft liegt benfalls cin wesentlicher Unterschied gegen dic :uropäischen Verhältnisse. Eine zweite Schwierigkeit ergibt sich für die amerikanischen Arbeiten lurch eine gewisse Konkurrenź verschiedener Organisationen. Tatsächlich wird die Gefahr, daß dieselben Arbeiten von zwei getrennten Ressorts doppelt ausgeführt werden, durch die Verständigung der betreffenden Behörden in der Regel vermieden, wenigstens scheint dies in dem Verkehr. der Coast und Geodetic Survey und der Geological Survey der Fall gewesen zu sein, von denen die letztere oft die topographischen Aufnahmen derselben Gebiete zu bearbeiten hat. Im Gegenteil haben sich diese beiden Vermessungsinstitute gegenseitige Förderung und Unterstützung angedeihen lassen, indem die bureaukratische Abgrenzung der verschiedenen Verwaltungsgebiete in den Vereinigten Staaten wohl weniger ausgebildet ist, als in unserm Staatswesen. Da $B$ aber Schwierigkeiten rorgelegen liaben, geht aus einer Vorlage der National Academy von 1878 hervor, welche sümtliche Vermessungsbehörden, deren es zu der Zeit 5 gegeben $z$ u haben scheint, unter die Oberleitung der Coast and Geodetic Survey stellen wollte, ein Antrag, der vom Kongreb indes abgelehnt wurde.

Daß die Survey mit sehr reichen Mitteln und einom großen Stab ron Mitarbeitern rechnen kann, ist ein Vorzug, den sie vor den meisten Staatsinstituten der Alten Welt roraus liat. Auf diese Weise setzen die I.eistungen der amerikanischen Organisationen uns oft in Erstaunen; eine vergleichende Wertschïtzung dor Methoden und Ergrebnisse mit denen europäischer Unternehmungen liegt dem Zweck dieser Zeilen fern, doch dürfen wir darauf hinweisen, dal auch vielfach Männer dabei tätig gewcsen sind, die aus europäischen Staaten stammen; es sei nur an Hilgard erinnert, der, ein Deutseher von Geburt, nicht weniger als to Jahre hindurch seine Dienste der Survey weihte, die leider seine unzurerlässige Amtsführung als Superintendent beendigte. Der hervorragende Anteil, den die Sehweiz bei der Gründung der Const and Geodetic Survey gehabt hat, ist bei der Jahrhundertfeior dadurch zum Ausdruck gekommen, dall der Schweizer Gesandte in Washington als einziger Vertreter eines fromden Staates zugegen war und insbesondere Hassler's Verdienste würdigtc. Nicht ohne Interesse liest man im negenwärtigen Augenblick die philosophisch durchwehte Ansprache des Prüsidenten der Vereinigten Staaten, der als letzter der Festredner auftrat. Die mit zahlreichen Abbildungren und den Porträts der Superintendenten ausgestattete Festschrift enthält auber den Reden und Vorträgen verschiedener Gelehrten anch interessante geschichtliche Dokumente.

\section{Kleine Mitteilungen.}

Die Trockenprodukte der Kartoffel. Zehn Prozente der Kirtoffelernte gingen früher durch Fäulnis, Atmun $\delta$ und Keimen der Kartoffeln verloren. Deshalb spielt die Kartoffeltrocknung, welche sich rasch \% einer nelen Industrie entwickelt hat, in der gregenwärtigen Kriegszeit eine sehr bedeutende Rolle. Den Hauptnährwert der Kartoffel bildet die Stärke, welche im Mittel $18 \%$ derselben betriigt. Die 25 Prozente Trockensubstanz der Kartoffel setzen sich zusammen aus Stärke, Zucker, Rohfaser, Fett, Stickstoffsubstanzen (EiweiB und $A$ miden) und ische. Die Wassermenge beträgt demnach durchschnittlich $75 \%$. Bei der Herstellung der Trockenprodukte wird viel Wasser verdampft, indem die Trockenkartofieln nur $15 \%$ besitzen. Dadurch erhöht sich der Gehalt an Kohlehydraten (namentlich Stärke) auf 72 bis $77 \%$ und der niedere Eiweib. gehalt der Rohkartoffeln von 2 auf beiläufig $6 \%$. Deshalb wird durch Herstellung der Trockenprodukte nicht nur eine unbegrenzt lange Aufbewahrung der Kartoffel möglich, sondern es nimmt auch deren Nähr. wert infolge Anreicherung an Kohlehydraten und Liweib zu. - Vor der Trocknung werden die Rohkartoffeln sorgfaltig gewaschen und dann in Streifen, sogenannte Schnitzel, oder Scheiben geschnitten, wenn 